\title{
Estratificación social de Graffar-Méndez Castellano y bajo peso al nacer en un hospital de Venezuela
}

\author{
Graffar-Méndez Castellano social stratification and low birth weight in a \\ hospital in Venezuela
}

\author{
Caterina Alessandra Squillaro-Rivero ${ }^{1}$, Carlos Miguel Ríos-González $\mathbb{D}^{2}$ \\ Ginno Alesssandro De Benedictis-Serrano $\mathbb{B}^{3}$
}

\author{
${ }^{1}$ Universidad Nacional Experimental Rómulo Gallegos, Venezuela \\ 2Universidad Nacional de Caaguazú, Facultad de Ciencias Médicas, Coronel Oviedo - Paraguay. \\ ${ }^{3}$ Universidad Carabobo Sede Aragua, Venezuela
}

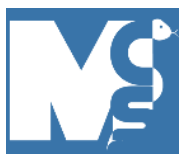

Recibido: $16 / 01 / 2021$

Revisado: 16/01/2021

Aceptado: 09/02/2021

\section{Autor correspondiente}

Carlos Miguel Ríos-González carlosmigue rios@live.com

\section{Conflictos de interés}

Los autores declaran no poseer conflictos de interés.

\section{Fuente de financiación}

Los autores no recibieron apoyo financiero para la investigación, autoría y/o publicación de este artículo.

Este artículo es publicado bajo una licencia de Creative Commons Reconocimiento 4.0 Internacional.

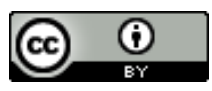

\section{Estimado editor:}

La Organización Mundial de la Salud (OMS) define el bajo peso al nacer como el peso menor que $2500 \mathrm{~g}$; la prematuridad y la restricción del crecimiento intrauterino (RCIU) son sus principales causas (1). El desarrollo fetal es un proceso basado en la proliferación y diferenciación de las células de manera armoniosa, por lo contrario, existen factores (maternos-fetales, placentarios, ambientales y sociales) que inciden de manera negativa en la formación óptima del feto el cual aumenta el riesgo de trastornos metabólicos o endocrinos en la vida adulta $(2,3)$.

Por todo lo anterior se planteó como objetivo describir la estratificación social Graffar-Méndez Castellano y el bajo peso al nacer en un hospital de Venezuela durante los meses de junio y julio del 2020, por lo que se realizó un estudio transversal, donde se aplicó un cuestionario donde se registró lo estratos socioeconómicos según el test de Graffar Mendez Castellanos (4) de las embarazadas que asistieron al servicio de obstetria del del Hospital Dr. José María Carabaño Tosta, Estado Aragua en Venezuela, durante junio y julio del 2020, para su posterior análisis con el peso de los recién nacidos.

Al estudio ingresaron 40 mujeres embarazadas, cuyos recién nacidos fueron 27 mujeres y 13 varones, de las cuales el $65 \%$ de los recién nacidos presentaron bajo peso al nacer; el $5 \%$ de las madres padecieron de alguna enfermedad durante la gestación, es importante mencionar que la minoría de las gestantes $30 \%$ aumento de peso durante el embarazo, con la aplicación del test de Graffar MendezCastellanos se evidencio que el $40 \%$ de las gestantes pertenecen al III estrato.

Al discriminar por los estratos socioeconómicos en los cuales encontraban las gestantes se encontró que el $16,66 \%$ de los neonatos en el estrato II tenían bajo peso, el $75 \%$ de los neonatos en el estrato III tenían bajo peso y el $100 \%$ en el estrato IV tenían bajo peso.

Es importante destacar las causas del bajo peso al nacer son multifactoriales: historias previas de bajo peso al nacer, infecciones vaginales, edad materna menor de 18 o mayor de 35 años, hábitos tabáquicos, habito alcohólicos, nuliparidad o multiparidad, bajo nivel educativo, también tiene implicaciones de índole social, familiar y económicas (5). 
Los resultados obtenidos en este estudio demuestran que el bajo nivel socioeconómico de las gestantes se observa con frecuencia en los recién nacidos con bajo peso, lo cual podría reflejar que a mayor precariedad podría aumentar el bajo peso al nacer. Por lo que motivamos a emprender nuevos estudios que enfoquen esta característica para mayores estudios debido a que actualmente es frecuente el bajo peso al nacer en los países en vías de desarrollo, a su vez con base en lo mencionado anteriormente se debe instruir a los profesionales de la salud sobre esta situación que puede ser prevenida si es notificada a tiempo, ya que se puede dificultar la captación y atención ante el auge del embarazo precoz y el desinterés por parte de las gestantes, siendo los entes gubernamentales quienes podrán actuar ante esto con las medidas correctivas adecuadas junto al personal de salud

\section{REFERENCIAS}

1. Daza V, Jurado W, Duarte D, Gich I, Sierra-Torres CH, Delgado-Noguera M. Bajo peso al nacer: exploración de algunos factores de riesgo en el Hospital Universitario San José en Popayán. Rev Colomb Obstet Ginecol 2009;60:124-34. https://doi.org/10.18597/rcog.337

2. Hales CN, Barker DJ. The thrifty phenotype hypothesis. Br Med Bull. 2001;60:5-20. https://doi.org/10.1093/bmb/60.1.5

3. Vaag AA, Grunnet LG, Arora GP, Brons C. The thrifty phenotype hypothesis revisited. Diabetologia. 2012;55:2085-2088. https://doi.org/10.1007/s00125-012-2589-y

4. Bauce Gerardo J, Cordova M. Cuestionario socioeconómicos aplicados a grupos familiares al distrito capital para investigaciones relacionadas con la salud pública. INHRR. 2010; 41(1):14-24. URL.

5. Paredes-Mondragón CV, Molano-Dorado H, Martínez-Gómez SY, Ortiz-Martínez RA, Arias-Linthon S, López-Benavides AC. Relación entre la ausencia de soporte social adecuado durante el embarazo y el bajo peso al nacer. Rev Colomb Psiquiat. 2019;48(3):140-148. http://dx.doi.org/10.1016/j.rcp.2017.11.002 